\title{
The effects of core stability strength exercise on muscle activity and trunk impairment scale in stroke patients
}

\author{
Seong-Hun Yu', Seong-Doo Park ${ }^{2, *}$ \\ 'Gwangju Trauma Center, Gwangju, Korea \\ ${ }^{2}$ Graduated School of Physical Therapy, Daejeon University, Daejeon, Korea
}

The purpose of this study was to examine the effects of core stability-enhancing exercises on the lower trunk and muscle activity of stroke patients. The control group $(n=10)$ underwent standard exercise therapy, while the experiment group $(n=10)$ underwent both the core stability-enhancing exercise and standard exercise therapy simultaneously. The standard exercise therapy applied to the two groups included weight bearing and weight shifts and joint movements to improve flexibility and the range of motion. The core stability-enhancing exercise was performed 5 times a week for 30 min over a period of 4 weeks in the room where the patients were treated. For all 20 subject, the items measured before the exercise were measured after the therapeutic intervention, and changes in muscle activity of the lower trunk were evaluated. The activity and stability of the core muscles were measured using surface electromyography and the trunk impairment scale (TIS). The mean TIS score and muscle activity of the lower trunk increased in the experiment group significantly after performing the core stability-enhancing exercise $(P<0.05)$. The results of this study show that the core stability-enhancing exercise is effective in improving muscle activity of the lower trunk, which is affected by hemiplegia.

Keywords: Core stability, Muscle activity, Stroke, Trunk impairment scale, Cerebral vascular accident

\section{INTRODUCTION}

Cerebrovascular accident (CVA) is a common nervous system disorder that occurs due to abnormal blood circulation in the brain with a completely developed nervous system (Forster et al., 2008). As the survival rate of patients with CVA increased owing to advances in medical technology, CVA became the most common internal cause of disablement (Barnett et al., 1999). In addition, CVA hinders the physical movements of daily life and causes dyspraxia and dysfunctions like cognitive impairment, dysesthesia, speech disorder, and visual impairment (Bobath, 1990). Unilateral paralysis accompanied by CVA reduces muscle control, body movements, and balance in unusual or asymmetrical positions. Thus, patients lose the ability to perform elaborate tasks and face difficulty while walking and standing up (Carr et al., 1985; Bobath, 1990). Moreover, most patients with unilateral paralysis have a tough time controlling their truncus while adjusting their posture.

The core is the biggest part of our body and plays an important role in the stabilization and movement of body segments. The truncus makes it possible for us to maintain posture and enables the movement of legs or arms, opposing gravity. It also contributes to smooth central movement so that our body easily can be changed to new posture (Ryerson et al., 2008).

In addition, the common problem of decrease in core muscle function, especially the external muscles, causes paralysis of the core muscle, decreases contraction, and increases the tendency to fall towards the paralyzed side, causing asymmetry. Because CVA reduces the quality of life by severely affecting balance and walking, which are the basic requirements for any physical activity, affected patients experience a huge sense of loss (Handa et al., 2000). Accordingly, physiotherapists should focus on dyspraxia and rehabilitation for balance and gait so that patients with brain damage can live an independent and functional life. Since long, the prognosis of CVA has been determined according to the part and size

${ }^{\star}$ Corresponding author: Seong-Doo Park

Graduated School of Physical Therapy, Daejeon University, 62 Daehak-ro, Dong-gu, Daejeon 300-820, Korea

Tel: +82-10-9215-2051, Fax: +82-63-540-5161, E-mail: psdbye@hanmail.net

Received: May 10, 2013 / Revised: June 7, 2013 / Accepted: June 14, 2013

This is an Open Access article distributed under the terms of the Creative Commons Attribution Non-Commercial License (http://creativecommons.org/licenses/by-nc/3.0/) which permits unrestricted non-commercial use, distribution, and reproduction in any medium, provided the original work is properly cited. 
of lesion. However, since the concept of brain plasticity became known, physicians are aware that the patients' degree of recovery can differ according to the quality of the rehabilitation program (Bach-y-Rita, 1981).

Core stability is usually used to strengthen the muscles around the abdominal, lumbar, and pelvic regions, because the muscles of these regions play an important role in stability as well as in controlling the lumbar posture by using tonic or postural muscles during whole-body exercises (Marshall and Murphy, 2005). The following muscles are related to core stability: multifidus, transversus abdominis, external/internal oblique abdominis, paraspinalis, gluteus, diaphragm in rear part, and hip muscles. The ventral muscles, multifidus, transversus abdominis, and oblique abdominis, provide core stability via cooperative contraction before moving out. The multifidus muscle serves as the intersegmental muscle placed on spiral part, followed by the interspinales and intertransverse muscles. These muscles control movement of the spinal units while lifting things and while rotating the core. Additionally, owing to the short length of these muscles, the reaction time is very rapid and this is highly important for maintaining stability (Kim and Kwon, 2001). Although muscles related to core stability have individual roles, they function in concert via cooperative contraction to establish core stability (Richardson et al., 1995). Core stability is a prerequisite for maintaining the proper posture of the lumbar and pelvic regions during sports activities. Exercises for core stability serve as treatment for simultaneously activating the abdominal and multifidus muscles in order to stabilize the body and head during the beginning of limb movements and during the course of these movements (Hodges and Richardson, 1997). The cooperative contractions of transversus abdominis and multifidus muscles improve the stability of each part when the spine is in neutral position or in motion (Porterfield et al., 1998). Patients with CVA lack selective movement control and thus the order of muscle movement is changed. These patients thus move in an unusual pattern, which results in much waste of energy and malfunctioning movement pattern. Although patients with CVA need to rebuild core stability in order to attain proper postures of the lumbar and pelvic regions during sports activities, most researches are conducted on athletes or patients with backache; research on patients with CVA is scarce (Rasmussen et al., 2003). Thus, in this study, we compare the core muscle activity of patients with CVA-induced hemiplegia before and after treatment for improving core stability. We also estimate the change in core muscle activity by using surface electromyography and the trunk impairment scale, and provide baseline data for core stability rehabilita- tion programs.

\section{MATERIALS AND METHODS}

\section{Patients}

Eighteen patients undergoing treatment for CVA-induced hemiplegia at a hospital located in Gwangju, Jeolla Province, Korea were enrolled. The patients were selected regardless of use of assisting devices like canes. However, the following eligibility criteria were considered: ability to walk for more than $32 \mathrm{ft}$, duration of disorder $>6$ months, do not have any problem in musculoskeletal model, absence of a cardiac disorder, complete understanding of this research, and ability to communicate. All patients signed an informed consent form. The patients' characteristics, objective medical information, and medical records were analyzed. The participants were divided into two groups: a control group of $10 \mathrm{pa}-$ tients who underwent kinesiatrics and an experiment group of 10 patients who participated simultaneously in a core stability-enhancing program and kinesiatrics (Table 1). In both groups, kinesiatrics was employed to not only teach patients how to support and move weights but also improve their flexibility and movable range of joint via joint exercises.

\section{Interventions}

The core stability-enhancing exercises were performed 5 times a week for a period of 4 weeks. Each participant exercised for 30 $\mathrm{min}$ in the room where they were being treated. The preliminary medical records of 18 participants were analyzed, and based on this, we evaluated the patients' muscle activity. The core stability-enhancing program was performed as follows. All core stability-enhancing exercises were preceded by reducing lumbar lordosis while the patient was lying rightly on an adjustable treatment table. After extending the hip and knee joints, both the hip and knees were supported by a pillow to maintain this posture. Additionally, to compensate for the action of the flexor muscle, the hip joint was blocked in advance by keeping the legs in a relaxed position. Next,

Table 1. Characteristics of subjects

\begin{tabular}{lcc}
\hline Group & CSG $(n=10)$ & CG $(n=10)$ \\
\hline Age $(\mathrm{yr})$ & $50.00 \pm 5.53$ & $52.64 \pm 4.56$ \\
Height $(\mathrm{cm})$ & $165.71 \pm 8.65$ & $165.45 \pm 5.00$ \\
Weight $(\mathrm{kg})$ & $65.29 \pm 14.18$ & $64.82 \pm 9.36$ \\
Onset (month) & $25.85 \pm 9.99$ & $30.96 \pm 7.67$ \\
Affected side (right/left) & $2 / 5$ & $5 / 6$ \\
\hline
\end{tabular}

CSG, core stability group; $\mathrm{CG}$, control group; $\mathrm{M} \pm \mathrm{SD}$, mean \pm standard deviation. 
the blade bone was retracted such that the shoulder girdle is positioned in abduction, and a towel was placed below the blade bone to prevent the pectoralis major from performing a compensatory action via relaxing both shoulders. Another preparatory step is enhancing the stability of the neck region. For this, the head was lifted and held in this position by flexing the abdominal region. At the same time, the neck was pulled down to prevent the column from bending. In addition, the multifidus and flexor muscles were contracted simultaneously. Maintaining this posture, the upper part of the back was lifted as much as possible and twisted slightly in a diagonal direction so that the right hand can face the left knee. This position was maintained for a moment before lowering the back. At this moment, the left arm was aligned, and therapists lead them in right direction and provide minimum help for patients who have difficulty in doing it due to weak abdominal muscle in order that they can control it by themselves.

This exercise was repeated; only this time the left hand faced the right knee for enhancing the abdominal muscles on the left. While maintaining this position, the jaw should be on the middle of the chest, and care should be taken that the jaw is not twisted. All these exercises enhanced the stability of core muscles. Particularly, the transversus abdominis and oblique muscles are strengthened when the multifidus and abdominal muscles are simultaneously contracted (Lee, 2007).

\section{Surface electromyography}

To measure the activity of the rectus abdominis, internal oblique, and erector spinae muscles, the Bagnoli EMG system (Delsys Inc., Boston, MA) with eight surface electrodes and one ground electrode was used. The sample rate of signal was set at $1,024 \mathrm{~Hz}$, and the notch filter (range, 20-450 Hz) at $60 \mathrm{~Hz}$ was used. The collected signs were analyzed using the Acquisition and Analysis Software after full-wave rectification. To check the activity of the musculus rectus abdominis, external abdominal oblique, internal abdominal oblique, and erector spinae muscles, all of which are paralyzed in patients with CVA, every muscle was attached to an electrode and highlighted by pen marking (Cram et al., 1998). To assess core stability, the conduction electrodes were placed on the regions of the muscle belly clearly visible during maximum muscular contraction. To reduce skin resistance to a surface electromyogram signal, the skin was washed with alcohol used for disinfection. The surface electrodes were attached to the skin by applying a small quantity of electrolyte gel. The ground electrode was attached to the 10th thoracic vertebra. For assessing the activity of the musculus rectus abdominis, the electrode was attached $5 \mathrm{~cm}$ above the belly button; for the external abdominal oblique, it was placed between the belly button and the ASIS; for the internal abdominal oblique, it was placed at a point approximately $2 \mathrm{~cm}$ from both sides. To normalize the action potential of the erector spinae muscle, activity on the electromyogram was measured at maximal voluntary isometric contraction while maintaining the core stability posture. After linear filtering data value during $10 \mathrm{sec}$, it was measured at average value during 8 sec except for first and last 1 sec.

\section{Trunk impairment scale (TIS)}

The TIS was used to measure the ability to control the core muscles. The scale of core damage that is clinical method for damage of core shown to CVA patient measure coordinated abilities of core as well as moving and statistically equilibrium at sitting position. The TIS has 17 provisions: static equilibrium, 3; dynamic equilibrium, 10; core's coordinated abilities, 4 . The TIS score ranges from 0 to 23, with higher points indicating good core control ability. Inter-tester reliability ranged from 0.87 to 0.96 and intra-tester reliability from 0.85 to 0.99 , indicating high reliability and internal validity (Verheyden et al., 2007).

\section{Statistical analyses}

To determine the effects of core stability-enhancing exercises, activity of the rectus abdominis, external and internal abdominal oblique, and erector spinae muscles, and core control ability of CVA patients were measured before and after performing core stability-enhancing exercises. All data are reported as mean (M) and standard deviation (SD). In addition, paired sample t-test was conducted according to the characteristics of the research variable. For differences within groups, independent samples t-test was performed. Significance was set at the level of 0.05. All statistical analyses were performed using Statistical Package for the Social Science (SPSS) Version 12.0

\section{RESULTS}

\section{Changes in muscle activity after core stability exercise}

According to the sample t-test, the difference in muscle activity before and after performing core stability-enhancing exercises was not statistically significant $(P<0.05)$. Table 2 shows the difference in the activity of each core muscle before and after performing core stability exercises for both experiment and control groups. The t-test was also used to investigate differences among groups. Intra-group differences were found after the exercise, not before $(P<0.05$, Fig. 1). 


\section{Changes in TIS score after core stability exercise}

Significant differences were seen in the TIS scores of the experiment group, and not the control group, before and after core stability-enhancing exercise $(P<0.05$, Table 2$)$. In addition, the t-test revealed intra-group differences after the exercise, but not before $(P<0.05$, Fig. 2$)$

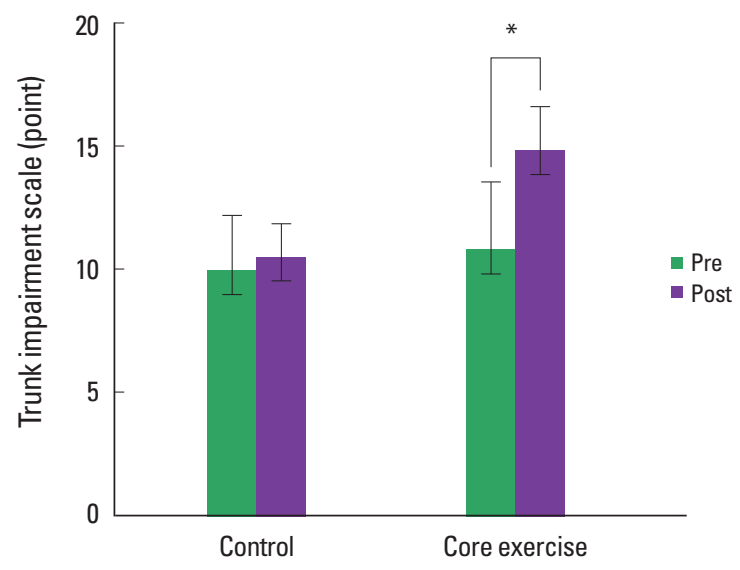

Fig. 1. Change in the TIS score of the core exercise group and control group $\left({ }^{*} P<0.05\right)$

\section{DISCUSSION}

This study evaluated the effects of a core stability-enhancing exercise program on the activity and stability of the core muscles of CVA-induced hemiplegic patients.

In rehabilitation therapy for CVA patients, postural control is necessary for smooth functional and voluntary movements while maintaining vertical posture (Johansson and Magnusson, 1991). Among various postural controls, core stability plays a major role in maximizing function and minimizing weight bearing at the joints (Kibler et al., 2006) while doing various activities like walking, running, and throwing. In addition, core stability is a highly reliable important predictive factor for the recovery of normal life activity and skilled tasks, which require the smooth movements of legs and arms (Hsieh et al., 2002; Feigin et al., 1996). According to a study by Massion (1994), there are two important functions of postural control system: (i) maintaining balance and resisting gravity and (ii) fixing one's position, which is used as datum spot, with respect to the environment. Postural control precedes movements of the arms and legs. Postural control aids the

Table 2. Muscle activity and TIS score before and after the core stability-enhancing exercise

\begin{tabular}{|c|c|c|c|c|c|c|}
\hline \multirow{2}{*}{ Muscle } & \multicolumn{2}{|c|}{ Control group $(n=10)$} & \multirow{2}{*}{$\mathrm{t}$} & \multicolumn{2}{|c|}{ Core excercise group $(n=10)$} & \multirow{2}{*}{$\mathrm{t}$} \\
\hline & Pre & post & & pre & post & \\
\hline Rectus abdominalis (mV) & $2.73 \pm 1.06$ & $2.93 \pm 1.19$ & 0.104 & $2.31 \pm 1.02$ & $4.32 \pm 2.42$ & $-1.944^{*}$ \\
\hline External oblique (mV) & $2.38 \pm 1.04$ & $2.50 \pm 0.98$ & 0.167 & $2.71 \pm 0.87$ & $4.90 \pm 2.51$ & $-3.367^{*}$ \\
\hline Internal oblique (mV) & $2.21 \pm 0.99$ & $2.01 \pm 0.97$ & 1.255 & $2.07 \pm 0.99$ & $5.27 \pm 3.33$ & $-2.909^{*}$ \\
\hline Erector spine (mV) & $1.73 \pm 0.71$ & $1.68 \pm 0.76$ & 0.167 & $1.76 \pm 0.98$ & $4.71 \pm 2.64$ & $3.357^{*}$ \\
\hline TIS (point) & $10.00 \pm 2.16$ & $10.50 \pm 1.35$ & -1.047 & $10.86 \pm 2.67$ & $14.86 \pm 1.77$ & $-6.481^{*}$ \\
\hline
\end{tabular}

${ }^{*} P<0.05 . M \pm S D$, mean \pm standard deviation; TIS, trunk impairment scale.

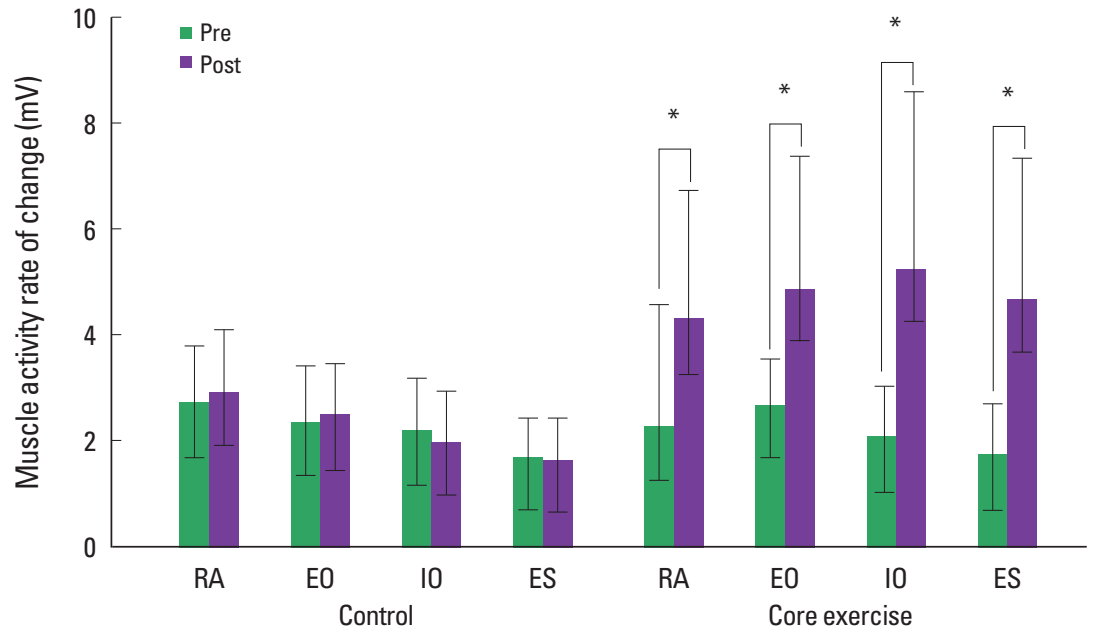

Fig. 2. Changes in the muscle activity of the core exercise group and control group. ${ }^{*} P<0.05$. $\mathrm{RA}$, rectus abdominis; $\mathrm{EO}$, external oblique; 10 , internal oblique; ES, erector spinae. 
core of the body adapt to a new posture while performing a particular exercise by preparing the body for unstable postures while moving the arms and legs (Dickstein et al., 1986). However, in many cases, CVA patients face difficulty in controlling posture because the preceding core muscle activity does not occur.

During any movement of the core, the transversus abdominis muscle is the first to contract (Cresswell et al., 1994; Konin et al., 2003). The oblique abdominal muscle endows stability during extension and side flexion of the core. These muscles are classified as tonic and are associated with safety. The external oblique muscle, which is the largest muscle of the abdomen and a surface muscle, is responsible for anterior tilting and helps the core remain stable by fixing the position of the pelvis (Richardson et al., 1995). Paraspinal muscles are surface muscles located among the extensors iliocostalis and longissimus. These muscles assist the multifidus muscle, which maintains the spine in a neutral position during the various curvatures caused by movements of the abdominal muscles. The thoracolumbar fascia along with contraction of the surrounding muscles activates proprioception. In addition, the muscle fascia connects arms and legs, providing fundamental stability during core movements (Akuthota et al., 2004). According to Hodges and Richardson (1997), the stability of the spine maintained by simultaneous contraction of the abdominal and multifidus muscles is preceded by reaction of the legs and arm exercises takes a role of central nerve. Contraction of the transversus abdominis and oblique abdominal muscles contribute to the function of the core regardless of the direction of power. On the other hand, the multifidus and rectus abdominis muscles have different reaction times according to the direction of power. Panjabi (1992) reported that the multifidus and transversus abdominis muscles, which are deep-seated, are mainly involved in spine stability and suggested that double multifidus muscle support the constituent parts of the spine. In this study, we propose that increase in the effective value of amplitude of the abdomen and erector spinae activity along with increase in effective value of amplitude of the multifidus and transversus abdominis muscle. Accordingly, core stability-enhancing exercises that develop the transversus and multifidus muscles prompt the central nerve system to positively affect core muscle activity.

In this study, the rectus abdominis, external and internal oblique abdominal, and erector spinae muscles were chosen based on the outcome of the core stability-enhancing exercise test. The changes in the activity of these muscles were investigated before and after the exercise, and the experiment group showed significant differences in the core muscle activity $(P<0.05)$. Our findings suggest that a 4-week core stability-enhancing exercise program induced activation of the core muscles of patients with CVA-induced hemiplegia.

To verify the effects of core stability-enhancing exercise, core control ability was evaluated using TIS. The control group showed no significant differences in the TIS score but the experiment group did $(P<0.05)$. Significant difference was found in the TIS score of the two groups after the test, not before. Core stability exercise seemed to have affected core control ability. To investigate the effect of additional core exercises on core conduction, Verheyden et al. (2009) conducted traditional exercise therapy on the control group and made the experiment group participate in traditional exercise therapy as well as individual core exercises for 30 $\min 4$ times a week over a period of 5 weeks. They found that an increase in the ability of the experiment group patients to maintain the sitting position $(P<0.05)$. In addition, they suggested the inclusion of core stability exercises for improving balance and selective trunk movement in CVA patients.

According to Lee and Baek (2007), core stability-enhancing exercise is an effective treatment method for improving the ability to maintain stationary posture and balance after a dynamic posture.

This study was conducted on selective patients and thus had a small sample size. In addition, during the experiment, the patients' daily life activities, except for during the treatment time, could not be controlled. In the future, studies with larger sample sizes and more controlled experiments should be conducted to further confirm the effects of core stability-enhancing exercise. In addition, this study included only CVA patients. The inclusion of normal healthy individuals will help in developing a more effective training program for CVA patients. Taken together, we can say that core stability-enhancing exercise helps improve core muscle stability, which is decreased due to hemiplegia. Thus, effective programs must be developed for enhancing the stability and function of core muscles in CVA patients.

\section{CONFLICT OF INTEREST}

No potential conflict of interest relevant to this article was reported.

\section{REFERENCES}

Akuthota V, Nadler SF. Core strengthening. Arch phys Med Rehabil 2004; 85:86-92.

Bach-y-Rita P. Brain plasticity as a basis of the development of rehabilita- 
tion procedures for hemiplegia. Scand J Rehabil Med 1981;13:73-83.

Barnett HJ, Eliasziw M, Meldrum HE. Evidence based cardiology: Prevention of ischemic stroke. BMJ 1999;318:1539-1543.

Bobath B. Adult hemiplegia: Evaluation and treatment: 3rd. London, England: Heinemann Medical Book. 1990.

Carr JH, Shepherd RB, Nordholm L. Lynne D. Inverstigation of new motor assessment scale for stroke patients. Phys Ther 1985;65:175-180.

Cram JR, Kasman GS, Holtz J. Introduction to surface electromyography. Gaithersburg, Marland: Aspen publishers Inc. 1998.

Cresswell AG, Oddsson L, Thorstensson A. The influence of sudden perturbations on trunk muscle activity and intra-abdominal pressure while standing. Exp Brain Res 1994;98:336-341.

Dickstein R, Hocherman S, Pillar T Shaham R. Stroke rehabilitation. Three exercise therapy approaches. Phys Ther 1986;66:1233-1238.

Feigin L, Sharon B, Czaczkes B, Rosin AJ. Sitting equilibrium 2 weeks after a stroke can predict the walking ability after 6 months. Gerontology 1996;42:348-353.

Forster A, Szabo K, Hennerici MG. Machanisms of disease: Pathophysiological concept of stroke in hemodynamic risk zones--do hypoperfusion and embolism interact. Nat Clin Pract Neurol 2008;4:216-225.

Handa N, Yamamoto H, Tani T, Kawakami T, Takemasa R. The effect of trunk muscle exercises in patients over 40 years of age with chronic low back pain. J Orthop Sci 2000;5:210-216.

Hodges PW, Richardson CA. Contraction of the abdominal muscles associated with movement of the lower limb. Phys Ther 1997;77:132-142.

Hsieh CL, Sheu CF, Hsueh IP, Wang CH. Trunk control as an early predictor of comprehensive activities of daily living function in stroke patients. Stroke 2002;33:2626-2630.

Johansson R, Magnusson M. Human postural dynamics. Crit Rev Biomed Eng 1991;18:413-437.

Kibler WB, Press J, Sciascia A. The role of core stability in athletic function.
Sports Med 2006;36:189-198

Kim Suhn-Yeop, Kwon Jae-Hoak. Lumbar stability exercises using the sling system. J of Kor Acad Orth Manu Ther 2001;7:23-39.

Konin JG, Beil N, Werner G. Functional rehabilitation. Facilitating the serape effect to enhance extremity force production. Athl Ther Today 2003;8:54-56.

Lee Byoung-Hee, Baek Ji-Young. The effects of core stability training on static and dynamic balance of stroke patients. J Kor Spor Health 2007; 18:623-634.

Massion J. Postural control system. Curr Opin Neurobiol 1994;4:877-887.

Marshall PW, Murphy BA. Core stability exercises on and off a Swiss ball. Arch Phys Med Rehabil 2005;86:242-249.

Panjabi MM. The stabilizing system of the spine. Part I : Function, dysfunction adaptation, and enhancement. J Spinal Disord 1992;5:383-389.

Porterfield JA, Derosa C. Mechanical low back pain: perspecitves in functional anatomy. 2nd. Philadelphia: W.B. Saunders Company 1998.

Rasmussen-Barr E, Nilsson-Wikmar L, Arvidsson I. Stabilizing training compared with manual treatment in sub-acute and chronic low-back pain. Man Ther 2003;8:233-241.

Richardson CA, Jull GA. Muscle control-pain control. What exercises would you prescribe? Man Ther 1995;1:2-10.

Ryerson S, Byl NN, Brown DA, Wong RA, Hidler JM. Altered trunk position sense and its relation to balance functions in people post-stroke. J Neurol Phys Ther 2008;32:14-20.

Verheyden G. Nieuwboer A, Van de Winckel A, De Weerdt W. Clinical tools to measure trunk performance after stroke: asystematic review of the literature. Clin Rehabil 2007;21:387-394.

Verheyden G, Vereeck L, Truijen S, Troch M, Lafosse C, Saeys W, Leenaerts E, Palinckx A, De Weerdt W. Additional exercises improve trunk performance after stroke: a pilot randomized controlled trial. Neurorehabil Neural Repair 2009;23:281-286. 